(C) [2010] IEEE. Reprinted, with permission, from [Behrens, M., Shoudong Huang ; Dissanayake, G., Models for pushing objects with a mobile robot using single point contact, Intelligent Robots and Systems (IROS), 2010

IEEE/RSJ International Conference on, 18-22 Oct. 2010]. This material is posted here with permission of the IEEE. Such permission of the IEEE does not in any way imply IEEE endorsement of any of the University of Technology, Sydney's products or services. Internal or personal use of this material is permitted. However, permission to reprint/republish this material for advertising or promotional purposes or for creating new collective works for resale or redistribution must be obtained from the IEEE by writing to pubs-permissions@ieee.org. By choosing to view this document, you agree to all provisions of the copyright laws protecting it 


\title{
Models for pushing objects with a mobile robot using single point contact
}

\author{
Michael Behrens, Shoudong Huang and Gamini Dissanayake
}

\begin{abstract}
In many mobile robotic manipulation tasks it is desirable to interact with the robots surroundings without actually grasping the object being manipulated. This nonprehensile manipulation allows the robot to interact in situations which would otherwise be impossible due to size or weight. This paper presents the derivation of a mathematical model of an object pushed by a single point and sliding in the presence of friction where the dynamic effects of mass and inertia are significant. This model is validated using both numerical simulation and experiments and is subsequently used to investigate the behavior of the system as the input conditions are varied. The derived dynamic model is also compared with a kinematic approximation from literature, showing that under certain conditions, the motion of a pushed object is similar to the motion of a non-holonomic vehicle. Finally, the experimental findings are discussed and promising directions for further work are proposed.
\end{abstract}

\section{INTRODUCTION}

The ability to manipulate the human environment is an essential competency for "social" robots in the home that can ubiquitously help in everyday living. While the fundamental issues associated with mobile robotics such as localization and mapping have received significant attention in the past decade [1] and are now well understood, manipulation in unstructured environments is still seen as a significantly challenging task. This paper considers one of the simplest tasks one could imagine, "to push a piece of furniture with a mobile robot". Despite the apparent simplicity, there are many challenges that need to be overcome to accomplish this task.

The ability to manipulate its surroundings enables a robot to achieve some meaningful work in a human environment, however, different manipulation tasks will require different strategies. Many tasks which require fine manipulation and/or transfer between different support surfaces will require the object to be grasped and then repositioned using a pick and place operation. However, there are a number of benefits that make non-prehensile manipulation preferable to grasping in certain situations. Since the robot does not need to grasp the object, the system can make use of simpler manipulators or can continue to operate in a limited fashion if a gripper fails. It also allows a robot to manipulate objects which are too heavy, large or awkward to grasp and lift such as furniture.

This work is supported by the ARC Centre of Excellence programme, funded by the Australian Research Council (ARC) and the New South Wales State Government.

The authors are with Faculty of Engineering and Information Technology, University of Technology Sydney, Australia

M. Behrens michael.behrenseuts.edu.au

S. Huang shoudong.huangeuts. edu. au

G. Dissanayake gamini.dissanayake@uts.edu.au
Another benefit provided by non prehensile manipulation is the ability to control objects with more degrees of freedom than are available to the robot. As an example, a 2DOF robot (point translating in plane) is able to control the position and orientation of an object sliding on the plane in the presence of friction.

In the proposed scenario the aim is not to grasp the object, hence, manipulation needs to be achieved by controlling the magnitude and direction of the force between the robot and the object. The primary difficulty faced when utilizing nonprehensile manipulation is that the motion of the object is usually a complex function of both the environment and manipulator actions. Coupled with the irreversibility of the pushing action, as we cannot simply reverse the motion and pull the object in the opposite direction, it is necessary to apply some form of trajectory planning to the activity to ensure that it is possible to reach the desired goal. As a result, it is essential that the motion planning algorithm take into account the constraints of the non-prehensile "grasp" when developing the robot trajectories [2]. In particular computing the object rotation requires the planner to deal with the dynamic motion of the object unless one is simply interested in the direction of rotation [3].

\section{RELATED WORK}

There has been significant activity on non-prehensile manipulations, particularly in relation to part feeding in the manufacturing domain. Mason [3] was the first to propose a simple rule for determining the rotation of an object when pushed by a flat fence. This requires knowledge of the object geometry, the pushing location and the center of friction of the support surface. Akella, Mason and Lynch [4], [5] went on to develop open loop pushing plans to reduce the orientation and position uncertainty of a sliding part, in order to guarantee that a part may be fed into the system in any orientation and will exit in a known configuration. This is particularly suited to the task of parts feeding in industrial applications [6], [7]. The strengths of this methodology are that suitable margins of error may be included so that the effect of support uncertainty is not significant in the determination of the object motion. This allows open loop plans which are guaranteed to produce a successful result to be generated. These systems, however, have to be designed for a specific task and rely on a fixed object geometry which must be polygonal. If a different part needs to be manipulated then an entirely new sequence of pushes needs to be developed. Another feature of these open loop pushing plans is that they are usually far from optimal. 
To overcome the problem of predicting the part orientation, most early applications in the manufacturing domain focused on using a flat fence to manipulate polygonal parts. This allows certain 'stable pushes' [8] where the frictional mechanics of the system will cause a face of the object to align with the pushing fence thereby controlling its orientation. It is possible to string together a number of stable pushes to perform open loop control. An analysis of the part geometry allows the reachable regions to be determined [9]. This method is limited by the need to have flat surfaces which are suitable for pushing and does not make use of the relative rotation between the robot and object for object positioning. Later work extended the flat fence idea to using a number of point contacts which create a virtual fence between the contact points and allows objects with curved or irregular surfaces to be manipulated.

Another technique which enables the system to compensate for angular rotation uncertainty is the manipulation of obstacles by coordinated teams of robots [10]. This also creates a "virtual fence" between the contacts and kinematically constrains the motion of the sliding object. Once again a series of stable pushing commands can be generated to manipulate an object to a desired goal configuration. The significant challenges in this field are related to the task of coordinating and communicating between the robots involved, deciding how decentralized control strategies can be derived and the extent of the information that needs to be communicated between the robots to accomplish desired behavior.

When the inertial effects are negligible and hence the motion of the object is dominated by the support friction, Kurisu and Yoshikawa [11] hypothesized that an object pushed at a single point will eventually reach a limiting angular velocity which is a function of the pushing angle, making the object behave in a manner similar to a nonholonomic vehicle. A validation that the kinematic model presented approximates well to the real behavior was not presented. However, in [12], the utility of this approximation was demonstrated through an experimental evaluation of a trajectory following technique for single point pushing. This is a surprising result, given that the notion of constant angular velocity under pushing is somewhat counter-intuitive, and given the observation by Mason [3] that without a dynamic analysis, only the direction of rotation can be computed.

Recently Igarshi et.al. [ref] presented a simple method for pushing an object to a desired goal location. Knowing the global position of both the object and goal they generate a set of paths, based on a simple dipole model, for the robot to follow. The nature of these paths is such that the robot will tend to push the object towards the goal location regardless of the relative size robot and object. One of the key benefits of this control rule is that it smoothly transitions into error recovery as the object begins to veer of course negating the requirement for a explicit recovery action. The authors conclude by indicating that this algorithm has the potential to be improved to be applicable in situations where rapid manipulation is required as well as situations where the object is pushed by a non-holonimic robot.

In the following sections a complete dynamic model of an object being pushed using a single point contact is derived. It is demonstrated that under some conditions the assumptions of Kurisu and Yoshikawa can be justified, potentially paving the way to developing simple trajectory planning techniques and control algorithms for single point pushing. The results of numerical simulations and experimental validation are presented and possible directions for the application of this model and other further extensions are discussed.

\section{ANALYSIS}

\section{A. Dynamic Model}

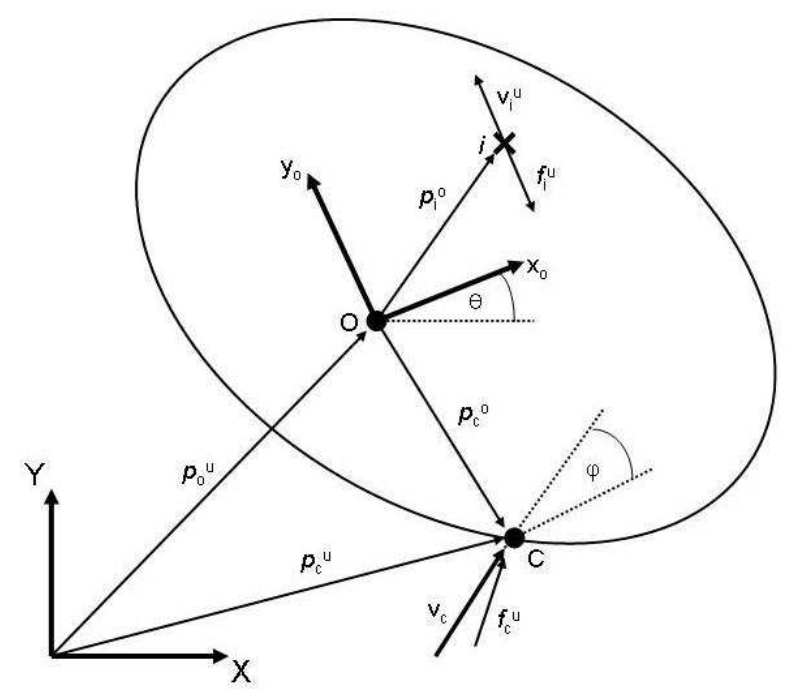

Fig. 1. A sliding object subject to a point force $v_{c}$ redraw this so that the contact point in on the object $\mathrm{x}$ axis

To obtain a model with behavior representing the moving system as close to true as possible the dynamics of the system must be considered. A list of notations used in the derivation is given in Table I. As in [11], it is assumed that the support distribution can be approximated to a finite set of known support points and that the magnitude of the friction force, the support geometry, mass and inertia are known and fixed. It is assumed throughout the analysis that all support points are in motion at all times and that the friction conforms to Coulomb friction. If there are more than three support points the problem becomes statically indeterminate and hence it is impossible to calculate the normal reaction forces and associated friction forces. In these situations the pressure distribution is assumed to approximate a constant distribution allowing the friction forces to be calculated. It is further assumed that the object is being pushed by a robot with sufficient power to impart the required force while maintaining constant velocity along a piecewise constant velocity profile. Given the motion controllers present in commonly available mobile robot systems, this is a reasonable assumption. The pushing force will be determined by the motion of the body and the friction forces imparted by the object environment interface. The final assumption is that the contact is a single 
TABLE I

Notations Used in Dynamic and Kinematic Models

\begin{tabular}{|c|c|}
\hline$p_{c}^{u}$ & position of the contact point in the global frame \\
\hline$p_{o}^{u}$ & origin of the object coordinate frame in the global frame \\
\hline$\theta$ & rotation angle of the object coordinate frame in the global frame \\
\hline$p_{c}^{o}$ & position of the contact point in the object frame \\
\hline$p_{i}^{o}$ & position of the $i$ th support point in the object frame \\
\hline$f_{i}$ & magnitude of the friction force at the $i$ th support point \\
\hline $\bar{v}_{c}$ & magnitude of the pushing velocity \\
\hline$\phi$ & direction of the pushing velocity in the object frame \\
\hline$n_{\phi}^{O}$ & direction vector of the pushing velocity \\
\hline$m_{c}$ & moment of inertia about the pushing contact point \\
\hline$I_{c}$ & friction force vector at $i$ th support point in the global frame \\
\hline$f_{i}^{u}$ & velocity vector at $i$ th support point in the global frame \\
\hline$v_{i}^{u}$ & co-efficient of friction at $i$ th support point \\
\hline$\mu_{i}$ & normal reaction force at $i$ th support point \\
\hline$N_{i}$ & angular velocity of the sliding object \\
\hline$\omega$ & numb force vector in the global frame \\
\hline$f_{c}^{u}$ & number of support points \\
\hline$n$ &
\end{tabular}

point and that the contact friction is sufficient such that the contact point does not slide along the perimeter of the body. We acknowledge that some of the assumptions are unreasonable in a practical situation. However, the focus of this paper is on the understanding of the behavior of an object being pushed. Our long term intention is to develop control strategies that are robust to the violation of these assumptions.

An inertial reference frame is defined relative to the ground plane and an object coordinate frame is fixed at the center of mass and rotates with the object. The motion of the object coordinate frame and hence the motion of the object center of mass relative to the global frame is given by

$$
p_{o}^{u}=p_{c}^{u}-R_{o}^{u} p_{c}^{o}
$$

where $R_{o}^{u}$ is the rotation matrix given by

$$
R_{o}^{u}=\left[\begin{array}{rr}
\cos \theta & -\sin \theta \\
\sin \theta & \cos \theta
\end{array}\right]
$$

differentiating (1) we get

$$
\dot{p}_{o}^{u}=\dot{p}_{c}^{u}-\dot{\theta} R_{o}^{u} D p_{c}^{o}
$$

with

$$
D=\left[\begin{array}{rr}
0 & -1 \\
1 & 0
\end{array}\right]
$$

if no contact slip occurs between the pusher and the object

$$
\dot{p}_{c}^{u}=R_{o}^{u} \dot{p}_{c}^{o}=\bar{v}_{c} R_{o}^{u} n_{\phi}^{o}
$$
by

where $n_{\phi}^{o}$ is the direction vector of the pushing force given

$$
n_{\phi}^{o}=\left[\begin{array}{c}
\cos \phi \\
\sin \phi
\end{array}\right]
$$

combining (3) and (5) we obtain the velocity of the center of mass

$$
\dot{p}_{o}^{u}=\bar{v}_{c} R_{o}^{u} n_{\phi}^{o}-\dot{\theta} R_{o}^{u} D p_{c}^{o}
$$

differentiating (7) we obtain the acceleration of the center of mass

$$
\ddot{p}_{o}^{u}=\bar{v}_{c} \dot{\theta} R_{o}^{u} D n_{\phi}^{o}-\ddot{\theta} R_{o}^{u} D p_{c}^{o}-\dot{\theta}^{2} R_{o}^{u} D^{2} p_{c}^{o}
$$

While the angular velocity cannot be computed directly it is possible to calculated the angular acceleration and integrate over time to obtain the angular velocity. Considering the friction forces, inertial forces, and the inertial moment, taking a moment balance about the contact point and rearranging we obtain

$$
0=\sum_{i=1}^{n}\left(R_{o}^{u}\left(p_{i}^{o}-p_{c}^{o}\right) \times f_{i}^{u}\right)+m R_{o}^{u} p_{c}^{o} \times \ddot{p}_{o}^{u}-\ddot{\theta} I_{c}
$$

where $I_{c}$ is the moment of inertia about the contact point and $f_{i}^{U}$ is the friction force at the $i$ th support point given by

$$
f_{i}^{u}=\frac{-v_{i}^{u}}{\left\|v_{i}^{u}\right\|} \mu_{i} N_{i}
$$

where $v_{i}^{u}$ is the velocity of the $i$ th support point in the global frame given by

$$
v_{i}^{u}=\bar{v}_{c} R_{o}^{u} n_{\phi}^{o}+\omega R_{o}^{u} D\left(p_{i}^{o}-p_{c}^{o}\right)
$$

and

$$
\omega=\dot{\theta}
$$

Combining $(8-12)$ provides a complex function for $\ddot{\theta}$ which we will denote simply as

$$
\ddot{\theta}=\dot{\omega}=F(\phi, \omega, \theta)
$$

If we denote $p_{o}^{u}=[x y]^{T}, p_{c}^{o}=\left[x_{c}^{o} y_{c}^{o}\right]^{T}$, then the dynamic equations of the system can be written as

$$
\left\{\begin{array}{l}
\dot{x}=\bar{v}_{c} \cos (\theta+\phi)+\omega\left(x_{c}^{o} \sin \theta+y_{c}^{o} \cos \theta\right) \\
\dot{y}=\bar{v}_{c} \sin (\theta+\phi)-\omega\left(x_{c}^{o} \cos \theta-y_{c}^{o} \sin \theta\right) \\
\dot{\theta}=\omega \\
\dot{\omega}=F(\phi, \omega, \theta)
\end{array}\right.
$$

To ensure that trajectories generated do not violate the capabilities of the pushing robot, the pushing force vector can be calculated from

$$
f_{c}^{u}=m \ddot{p}_{o}^{u}-\sum_{i=1}^{n} f_{i}^{u}
$$

Combining (8) and (15) yields

$$
f_{c}^{u}=m\left(\bar{v}_{c} \dot{\theta} R_{o}^{u} D n_{\phi}^{o}-\ddot{\theta} R_{o}^{u} D p_{c}^{o}-\dot{\theta}^{2} R_{o}^{u} D^{2} p_{c}^{o}\right)-\sum_{i=1}^{n} f_{i}^{u}
$$

This equation can be used to compute pushing force and determine if the capabilities of the robot or if the pushing angle limits have been exceeded allowing contact slip to occur, making it possible to test whether the relevant assumptions hold. 


\section{B. Kinematic Model}

In this section we re-present the kinematic equations obtained by Kurisu and Yoshikawa [11] for comparison. The key assumptions used in their analysis are that the net moment about the contact point is zero and effect of inertial forces due to the mass and the moment of inertia of object are negligible in comparison to support friction forces.

Writing an equation for moment balance about $C$, rearranging and simplifying yields

$$
\begin{aligned}
& 0=M_{f_{c}}^{o}=\sum_{i=1}^{n} \frac{B_{i}+\psi A_{i}}{\sqrt{1+2 \psi B_{i}+\psi^{2} A_{i}}} \bar{f}_{i} \\
& \text { with } \\
& A_{i}=\left(p_{i}^{o}-p_{c}^{o}\right)^{T}\left(p_{i}^{o}-p_{c}^{o}\right) \\
& B_{i}=\left\{D\left(p_{i}^{o}-p_{c}^{o}\right)\right\}^{T} n_{\phi}^{o}
\end{aligned}
$$

Since $A_{i}$ and $\bar{f}_{i}$ are known and $B_{i}$ is a function of $\phi$, we can see from above equation that $\psi$ is a function of $\phi$. If we denote this function as $\psi(\phi)$, then we have

$$
\dot{\theta}=\bar{v}_{c} \psi(\phi)
$$

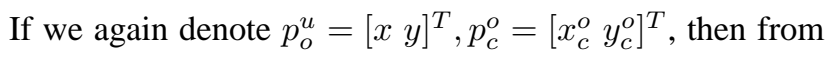
(7) and (18) we can get the equations of motion

$$
\left\{\begin{aligned}
\dot{x} & =\bar{v}_{c} \cos (\theta+\phi)+\bar{v}_{c} \psi(\phi)\left(x_{c}^{o} \sin \theta+y_{c}^{o} \cos \theta\right) \\
\dot{y} & =\bar{v}_{c} \sin (\theta+\phi)-\bar{v}_{c} \psi(\phi)\left(x_{c}^{o} \cos \theta-y_{c}^{o} \sin \theta\right) \\
\dot{\theta} & =\bar{v}_{c} \psi(\phi)
\end{aligned}\right.
$$

It is clear that the above equation is strikingly similar to the full dynamic equation given by (14). The real difference is that the dynamic equations demonstrate that the object angular velocity will evolve as a function of time as expected, while the kinematic equations state that angular velocity is only a function of the pushing angle. The question we aim to answer in the following section is whether the latter, exploited by Kusisu and Yoshikawa in their motion planning and control algorithms, is reasonable and if so under what conditions.

\section{NUMERICAL SIMULATION}

Matlab was used to implement and solve the equations of motion derived in the previous section. The simulated object is a flat square plate, $250 \mathrm{~mm}$ along the side with a support in each corner. The object is pushed at one of the object corners at a constant velocity. With the mass selected to be $1 \mathrm{~kg}$, assuming a uniform mass distribution, the moment of inertia about the pushing point is $0.1667 \mathrm{~kg} / \mathrm{m}^{2}$. The ground reaction force is assumed to be equally distributed between the support points and a co-efficient of friction of 0.5 is assumed. Several input velocities with a constant magnitude varying from $0.2 \mathrm{~m} / \mathrm{s}$ to $1.0 \mathrm{~m} / \mathrm{s}$ are used. The object motion is simulated for piecewise constant input shown in Fig. 2. Note that the input of $\pi / 4$ radians directs the pushing vector through the center of friction and hence produces pure translation.

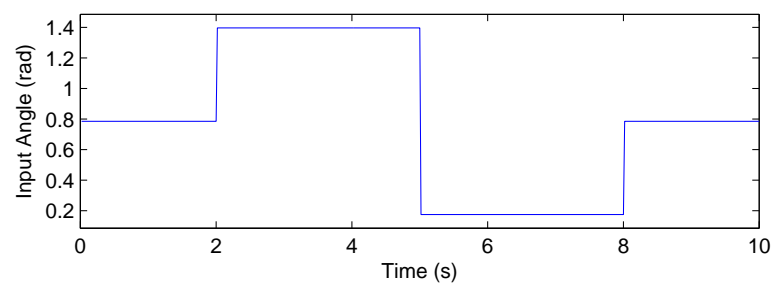

Fig. 2. Pushing velocity input angle $\phi$

The simulation results show that differences exist between the response of the dynamic model and the kinematic approximation as expected. The first notable difference is that the steady state angular velocity is lower in the dynamic model than is predicted by the kinematic approximation. The effect can be understood by considering the interaction of forces which cause the system to reach a steady state. If we make the assumptions listed earlier, the direction of a particular support friction force will be opposite to the instantaneous velocity of the support point while the magnitude will remain constant. As the instantaneous velocity of a support point is composed of the constant linear pushing velocity and the angular velocity crossed with the position vector for the support point, the support friction vector is actually a function of the angular velocity. If the angular velocity is momentarily lower than the steady state angular velocity then the direction of the friction reaction forces will change such that the resulting moment about the pushing point will act to increase the angular velocity. Conversely, if the angular velocity is momentarily higher the shift in the friction forces will act to reduce the angular velocity towards the steady state value. Hence, it can be seen that the system will reach a stable equilibrium motion if the input is kept constant and the locations of the support points do not change. For the kinematic approximation, only the support friction forces must be considered in the moment balance equation. However, for the dynamic case an additional force, the so called centripetal force due to the accelerating center of mass, must be included in the moment balance. It is logical that the presence of this additional force will cause the system to reach a different steady state. The nature of the centripetal force is such that its presence will always act to reduce the steady state angular velocity of the system. The centripetal force increases as the pushing velocity increases resulting in an greater deviation from the kinematic approximation as the pushing velocity increases.

Another effect which can be observed is a period of transient response as the angular velocity reaches the steady state value. This effect can be readily explained by the presence of rotary inertia in the dynamic model which prevents the objects angular velocity from changing instantly. Instead, the moment imbalance about the pushing point creates a torque which acts to accelerate the object towards the steady state angular velocity. The steady state velocity increases as the pushing velocity increases and hence the period of transient response increases in duration. Once the transient period has 
expired and the system has reached a steady state it is still be possible to use a non-holonomic vehicle model if a suitable scaling factor is selected.

For slow pushing speeds such as the one shown in figure bla the trajectories predicted by both the kinematic and dynamic models correspond quite closely. This confirms that the kinematic approximation can successfully be applied when the quasistatic assumption is valid. As the pushing velocity increases the kinematic and dynamic responses will begin to diverge. Exactly when the kinematic approximation will cease to be suitable will depend on the application and on the controllers ability to correct for errors. Figure bla shows the response of the sliding object when pushed at $1 \mathrm{~m} / \mathrm{s}$ which is approximately a comfortable walking pace. At this speed the dynamics of the system are significant and the two effects mentioned in the preceding paragraphs introduce significant errors while the object is rotating. As there is no feedback in this simulation theses errors accumulate quickly and result in a vastly different final location.

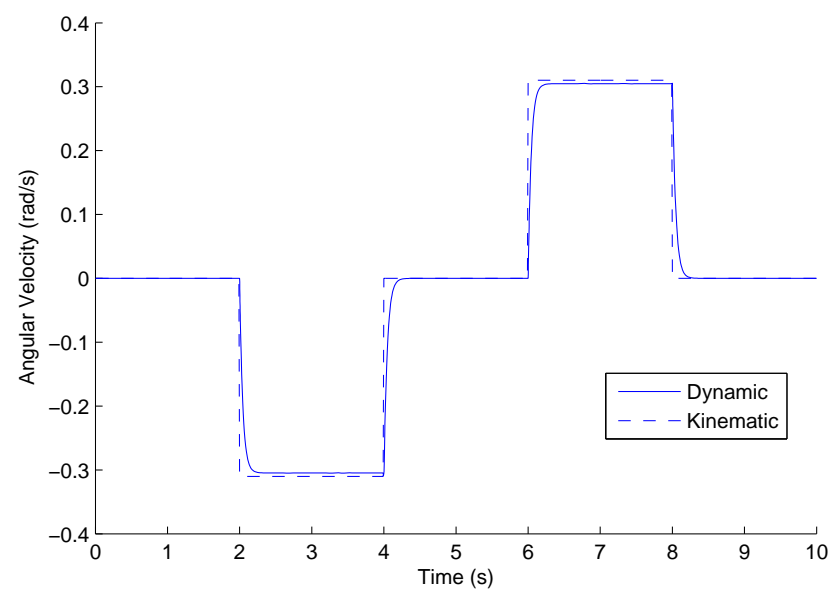

Fig. 3. Angular velocity response for a range of input velocities for Dynamic and Kinematic models

Similar effects can be obtained by reducing the co-efficient of friction to simulate the object sliding on a slippery surface such as a smooth concrete floor. By reducing the friction co-efficient the friction reaction forces are in turn reduced, placing a greater weight on the inertial effects of the object mass. From these results it appears that a higher pushing velocity is possible on rough or sticky surfaces if the quasistatic condition must be maintained.

It is curious to note that increasing the mass of the object did not change the behavior of the system as the moment of inertia and the friction forces are also increased resulting no net effect on the system response.

\section{EXPERIMENTAL VALIDATION}

To further investigate the dynamic model presented in this paper an instrumented test rig was developed. The test rig takes the form of a square plate with four lacquered wooden feet as support points. It has a variety of force torque

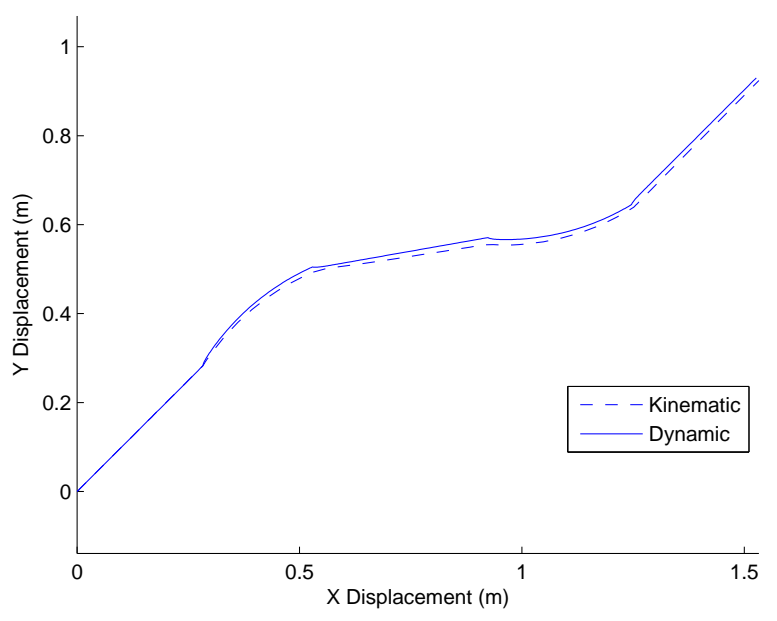

Fig. 4. $\mathrm{X}$ and $\mathrm{Y}$ displacements of the center of mass for Dynamic and Kinematic models with $\overline{v_{c}}=0.2$

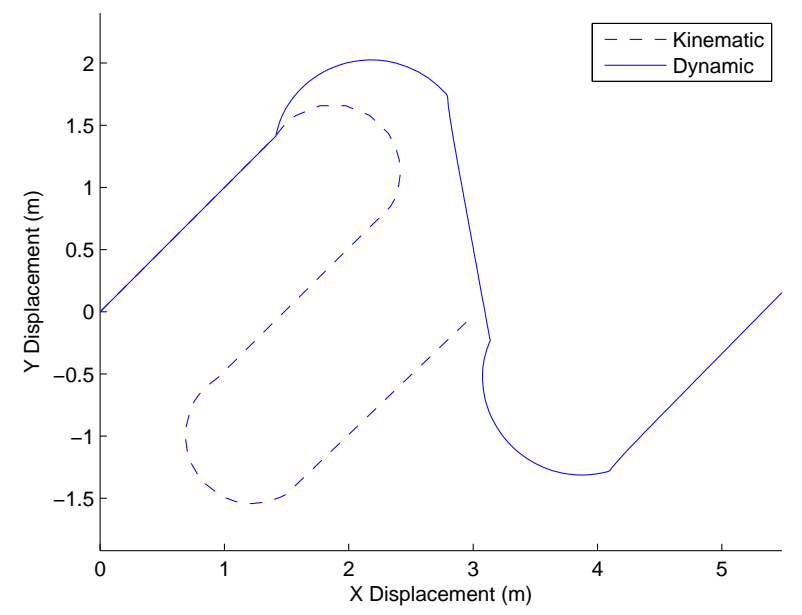

Fig. 5. $\mathrm{X}$ and $\mathrm{Y}$ displacements of the center of mass for Dynamic and Kinematic models with $\overline{v_{c}}=1.0$

sensors which allow the pushing force as well as the friction and normal forces at each support point to be measured. An overhead camera is used to track test rig motion and provides position and orientation information. Preliminary experiments have been conducted to date and the results are promising. The development of a online feedback system to fully validate the model is planned for the near future.

The first set of experiments where performed pushing the test rig by hand. This was done so that the input could be kept at a roughly constant angle without requiring a feedback control system. The limitation of pushing by hand is that the input was neither constant velocity or repeatable. However, some insight can still be gained by computing the ratio between the linear and angular velocities to normalize the results, shown in figure bla. The points to note are that the rise time for fast motion lasts for a number of frames where as the slow motion reaches the constant level within a single 
frame. Also note that the average velocity ration is lower for the rapid motion as predicted by the dynamic model.

Graph showing the results of the early pushed by hand experiment.

A second set of experiments was performed using a robot arm to apply a repeatable input motion. Since the input was open loop, the input velocity was maintained at constant angle relative to the ground plane rather than to the object as described in the model. Never the less, plotting the trajectories taken by the center of mass shows an increase in the turning radius i.e. a flatter trajectory as the velocity is increased.

Graph of the motion of the center of mass showing the increased radius of the path at higher speed

\section{DISCUSSION AND FUTURE WORK}

This paper presents a dynamic model for an object sliding in the presence of friction which is validated by both simulation and experimental results. It is demonstrated that under quasistatic conditions it is indeed possible to use a simple kinematic approximation similar to a non-holonomic vehicle model to describe the motion of an object pushed by a single point contact. It is also demonstrated however, that the kinematic approximation is not sufficient under situations where the dynamics of the system have a significant effect on the system response. In these situations it was found that the angular velocity will experience a transient period before reaching a steady state lower than is predicted by the kinematic model. The general character of the system response, when the pushing angle is constant relative to the object, appears to follow the behavior of a first order system. This may explain why people find it relatively easy to manipulate objects by pushing even when the object ground conditions such as friction and the load distributions rapidly change.

A model of the dynamic behavior of a rapidly pushed object can be used in a number of future research directions. One potential application is to enhance existing path planning algorithms to allow feasible trajectories for both the robot and object to be developed for rapid manipulations. It may also be incorporated into more efficient control strategies such as model predictive control. Another related area which may be interesting for future investigation is the task of performing online friction estimation to predict the magnitude and location of a resultant friction force in real time, which could be passed through to an improved feedback controller. The next step we are planning to take is to undertake further investigation of the dynamic behavior of the system. It is hoped that an algorithm for calculating the appropriate scaling factors to relate the dynamic and quasistatic conditions, as well as methods for calculating them from a minimal data set will be developed.

\section{REFERENCES}

[1] H. Durrant-Whyte and T. Bailey, "Simultaneous localisation and mapping (slam): Part i the essential algorithms," IEEE ROBOTICS AND AUTOMATION MAGAZINE, vol. 13, no. 2, pp. 99-108, 2006.

[2] K. M. Lynch, Nonprehensile robotic manipulation: controllability and planning. PhD thesis, Pittsburgh, PA, USA, 1996.

[3] M. T. Mason, "Mechanics and planning of manipulator pushing operations," Int. J. Rob. Res., vol. 5, no. 3, pp. 53-71, 1986.

[4] S. Akella and M. T. Mason, "Posing Polygonal Objects in the Plane by Pushing," The International Journal of Robotics Research, vol. 17 no. 1 , pp. 70-88, 1998 .

[5] K. Lynch, "Locally controllable manipulation by stable pushing," Robotics and Automation, IEEE Transactions on, vol. 15, pp. 318 -327 , apr 1999.

[6] S. Akella, Robotic manipulation for parts transfer and orienting: mechanics, planning, and shape uncertainty. $\mathrm{PhD}$ thesis, Pittsburgh, PA, USA, 1996. Adviser-Mason, Matthew T.

[7] S. Akella and M. Mason, "Parts orienting by push-aligning," in Robotics and Automation, 1995. Proceedings., 1995 IEEE International Conference on, vol. 1, pp. $414-420$ vol.1, 21-27 1995.

[8] K. M. Lynch and M. T. Mason, "Stable pushing: mechanics, controllability, and planning," Int. J. Rob. Res., vol. 15, no. 6, pp. 533-556, 1996.

[9] K. Lynch and M. T. Mason, "Controllability of pushing," IEEE International Conference on Robotics and Automation, vol. 1, pp. 112 119, 1995.

[10] Q. Li, Coordinated nonprehensile manipulation for parts transfer: Mechanics, control, and planning. $\mathrm{PhD}$ thesis, School of Engineering Science, Simon Fraser University, 2006.

[11] M. Kurisu and T. Yoshikawa, "Trajectory planning for an object in pushing operation," in Japan-U.S.A. Symposium on Flexible Automation, (Kobe, Japan), pp. 1009-1016, 1994.

[12] M. Kurisu and T. Yoshikawa, "Tracking control for an object in pushing operation," Journal of Robotic Systems, vol. 14, no. 10, pp. 729-739, 1997. 